ARTÍCULO: HACIA UN DISEÑO DE POLÍTICAS PÚBLICAS DE DATOS ABIERTOS DE MEDIO AMBIENTE

\title{
HACIA UN DISEÑO DE POLÍTICAS PÚBLICAS DE DATOS ABIERTOS DE MEDIO AMBIENTE
}

Towards a design of public policies on open data on the environment

Rumo a um desenho de políticas públicas de dados abertos sobre o meio ambiente

\section{RESUMEN}

El presente trabajo tiene como fin proponer el reconocimiento de las políticas públicas de datos abiertos de medio ambiente, no de los datos en sí ni de las bases de datos gubernamentales, sino de la existencia de una dinámica complementaria entre las políticas públicas de medio ambiente, los datos abiertos de medio ambiente y las políticas públicas de datos abiertos. Estas últimas políticas hasta ahora o son inexistentes o incipientes o poco confiables, a pesar de su peso en la sostenibilidad y el futuro de cualquier sociedad y economía.

PALABRAS CLAVE: datos abiertos, políticas públicas, medio ambiente, sostenibilidad, desarrollo

\section{Martin Cutberto Vera Martínez ${ }^{1}$}

martin.vera@uabc.edu.mx

ORCID: http://orcid.org/0000-0002-3415-9357

\section{María Concepción Martínez Rodríguez²}

mcmartinezr@ipn.mx

ORCID: http://orcid.org/0000-0003-3094-5411

1 Universidad Autónoma de Baja California, México

2 Instituto Politécnico Nacional, México

Sometido 07-11-2019 Aprobado 05-04-2020

Evaluado por el sistema double blind review.

DOI: http://dx.doi.org/10.12660/cgpc.v25n82.80506 


\section{ABSTRACT}

This work aims to propose the recognition of public policies on open environmental data, not on the data themselves or on government databases, but on the existence of complementary dynamics between public environmental policies, open environmental data and open data public policies. So far, these latest policies are either nonexistent or incipient or unreliable, despite their weight on the sustainability and the future of any society and economy.

KEYWORDS: open data, public policies, environment, sustainability, development

\section{RESUMO}

O objetivo deste artigo é propor o reconhecimento de políticas públicas de dados ambientais abertos, não dos dados em si ou das bases de dados governamentais, mas da existência de uma dinâmica complementar entre as políticas públicas de meio ambiente. Ambiente de dados abertos e políticas públicas de dados abertos. Até agora, essas políticas são inexistentes, incipientes ou não confiáveis, apesar de pesarem na sustentabilidade e no futuro de qualquer sociedade e economia

PALAVRAS-CHAVE: dados abertos, políticas públicas, meio ambiente, sustentabilidade, desenvolvimento

\section{INTRODUCCIÓN}

Es importante mencionar que este trabajo no se basa en el análisis estadístico de datos o de las bases de datos públicas o respecto de las reglas jurídicas para crearlas. Sino que, como se indica desde el título, se propone analizar la conceptualización, el contenido, el alcance y las perspectivas de creación de Políticas Públicas de Datos Abiertos en materia de Medio Ambiente, como un reto intelectual y administrativo urgente, frente al agravamiento de los problemas medio ambientales. Es decir, no se centra en los datos o los sistemas y la existencia de dichos datos, sino en la posibilidad, por su carácter transversal, de crear una política pública específica para los datos abiertos, para abordar su diseño, su operatividad y medir su impacto, recirculando su valor, para generar mayor utilidad social y pública. Con una perspectiva de sustentabilidad a largo plazo.

Como antecedente de su necesidad, señalamos el carácter global del problema de la degradación ambiental, que es un fenómeno en sí mismo y no sólo la consecuencia de otros como la economía, la industrializa- ción o el consumo. Hay evidencia científica suficiente para afirmar que la degradación de los recursos naturales y la baja efectividad de la atención social y pública, incrementan logarítmicamente la problemática.

En el caso de estudio de este artículo, que es México, tenemos como antecedente que en 1992, la Organización de las Naciones Unidas publicó un informe que reveló, luego de difundir los niveles de dióxido de azufre, partículas suspendidas, monóxido de carbono y ozono troposférico y compararlos con los niveles aceptables de la Organización Mundial de la Salud, los altos niveles de polución de la Ciudad de México (OMS y GEMS, 1992). Dicho informe arrojó datos, que suscitan la controversia del deterioro ambiental, mostrando que las directrices de la OMS no se cumplen y que la norma nacional de calidad del aire se excede ocasionalmente en algunos sitios específicos, impactando salud, cultura, servicios públicos, economía y calidad de vida.

El gráfico 1 muestra las tendencias de la calidad del aire en la Ciudad de México desde 1990 al 2016. 


\section{HACIA UN DISEÑO DE POLÍTICAS PÚBLICAS DE DATOS ABIERTOS DE MEDIO AMBIENTE}

Gráfico 1. Tendencias de la calidad del aire en la Ciudad de México, 1990-2016

Informe anual 2016

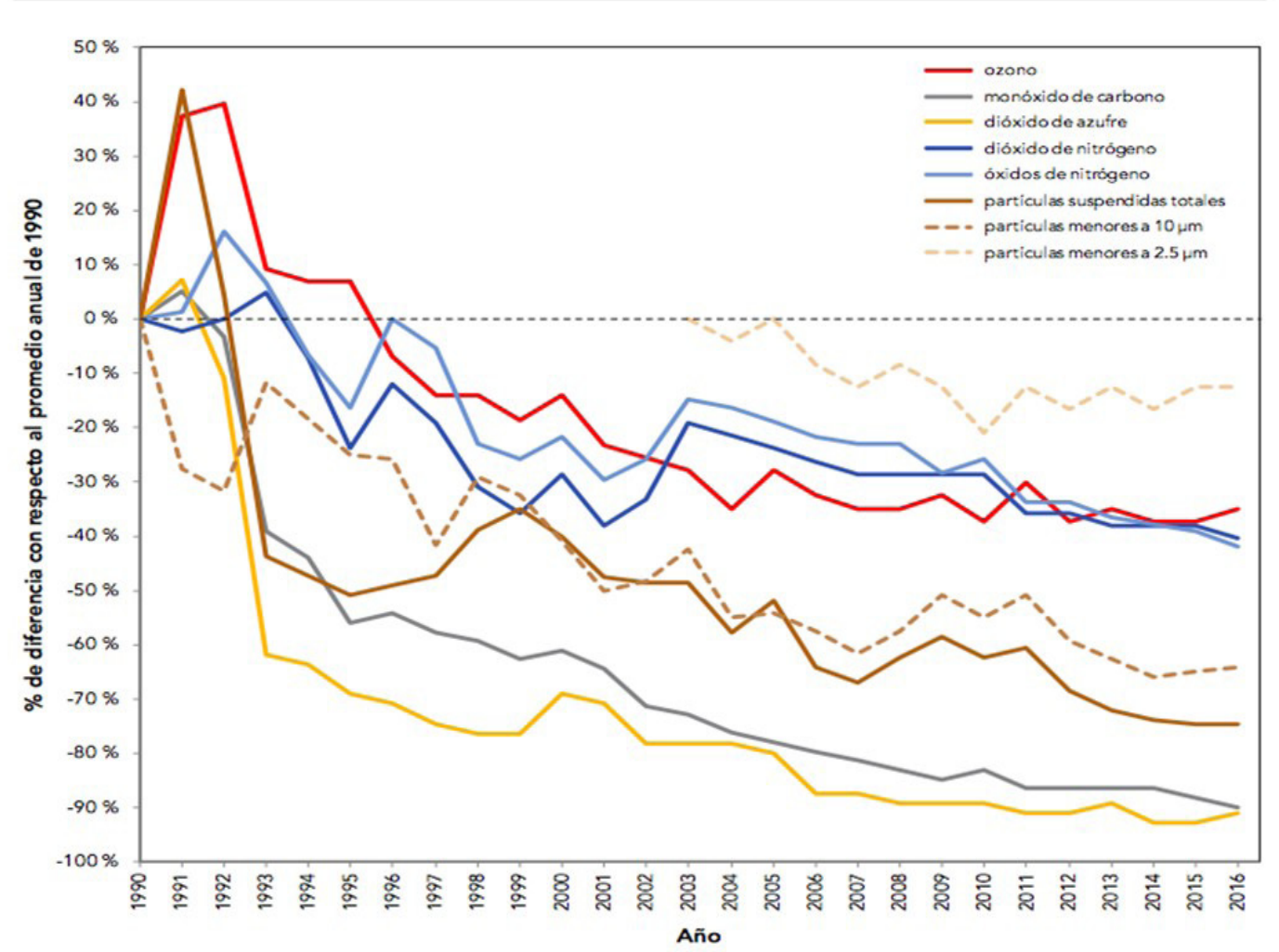

Recuperado de Secretaría del Medio Ambiente de la Ciudad de México. Calidad del aire en la Ciudad de México, informe 2016

El Informe se hizo extensivo a todas las actividades y a todo el territorio, lo que hizo necesario tener en cuenta el desarrollo económico y las tendencias futuras al considerar la relación funcional entre bienestar, consumo de energía y contaminación, y para esto necesita contar necesariamente con una Política Pública de Datos Abiertos, que sustente la toma de decisiones en cada sector nacional e internacional.

A partir de ese momento, fueron tomados en cuenta muchos datos sobre medio ambiente para visibilizar diferentes problemas. Si bien desde entonces el gobierno mexicano ha desarrollado políticas públicas am- bientales que tienen por objetivo mejorar la calidad de vida de sus habitantes, lo cierto es que muchas veces dichas acciones han sido llevadas a cabo de manera aislada 0 desvinculada de otras políticas de estado. Paralelamente en los últimos años - y dados los avances tecnológicos - el gobierno ha decidido crear acciones concretas para facilitar el acceso a la información pública, la protección de los datos y la transparencia. Así, se han desarrollado diferentes políticas públicas de datos abiertos que facilitan el acceso y la reutilización de los mismos. En ese sentido, hemos observado que, hasta la fecha, la investigación sobre políticas públicas ambientales no ha considerado su inte- 
rrelación con las políticas públicas de datos abiertos y de la misma manera, los datos abiertos han sido estudiados de manera general, sin tener en cuenta la singularidad e importancia de los datos abiertos de medio ambiente en la generación de políticas públicas concretas, que se sirvan de ellos.

Es por ello que el presente trabajo tiene por fin resaltar el aspecto complementario de las políticas ambientales, los datos abiertos de medio ambiente y las políticas de datos abiertos y, sobre todo cómo el resultado de dicha complementariedad, cuyo eje principal es el dato abierto de medio ambiente, da lugar a una propuesta analítica y de aproximación, de una nueva categoría de política pública, a saber, la política pública de datos abiertos ambientales.

Nuestra propuesta pretende iniciar un nuevo debate sobre el reconocimiento de la categoría de política pública de dato abierto de medio ambiente. Por este motivo consideramos que un primer abordaje debe ser descriptivo, y que, en este estado del arte, son necesarias determinadas propuestas de diseño respecto de esta nueva categoría de política pública. No se presenta a nivel de propuesta, porque se considera necesario en primer lugar, sentar su abordaje conceptual e iniciar su debate académico.

Por esa razón, el presente artículo se encuentra dividido en tres secciones: En primer lugar, se presentan los principales aspectos de los datos abiertos de medio ambiente en México. En segundo lugar, se describen las políticas públicas ambientales y las políticas públicas de datos abiertos. En tercer lugar, se resalta la necesidad de complementar las políticas públicas ambientales y las políticas públicas de datos abiertos para dar surgimiento a una categoría singular que será conceptualizada: la política pública de dato abierto de medio ambiente. Por último, presentaremos conclusiones y líneas de investigación para futuras indagaciones en la materia.

La metodología para realizar este trabajo consistió en contrastar los elementos en que coinciden diversos autores, que determinan la definición y el contenido de una política pública, partiendo de la hipótesis de que los datos abiertos en materia de medio ambiente, en este caso en México, no reciben ni el tratamiento ni el diseño de una política pública, si lo que se pretende buscar es generar un comportamiento diferente entre los actores que afectan el medio ambiente y también de aquellos que tienen como misión preservarlo. En consecuencia, fue necesario realizar un análisis documental y de tendencias institucionales, para verificar y comprobar dicho planteamiento. Como se podrá observar en la lectura, a pesar de la relevancia de los datos, de la legislación existente y de los sistemas administrativos creados, las bases de datos abiertos, así como su administración y acceso no configuran en sí, una política pública, por lo que resulta necesario abrir un debate interdisciplinario, interinstitucional e intrasocial, para, con un enfoque Bottom Up, generar la estructuración, primero conceptual y después metodológica e institucional en el camino de una política pública que hoy es urgente, ante el acelerado deterioro medio ambiental y ante la necesidad de definiciones democráticas e institucionales. Se siguió una lógica deductiva-inductiva entre la teoría, los fines públicos declarados y la práctica existente. Esperemos que la contribución de este trabajo sea relevante y de in- 
HACIA UN DISEÑO dE POLÍtICAS PÚBLICAS dE dATOS ABIERTOS DE MEDIO AMBIENTE

terés para la comunidad académica.

\section{MARCO TEÓRICOY EMPÍRICO}

Los datos abiertos y los datos abiertos de medio ambiente en México.

Hoy día, podemos encontrar un amplio número de iniciativas de datos abiertos en el mundo, orientadas por un objetivo común, que es la apertura de bases de datos que le permitan a la ciudadanía examinar y reutilizar la información pública, al amparo de reformas legales estandarizadas, con propósitos sociales, de participación ciudadana, empresariales, periodísticos o académicos. La OCDE (Organización para la Cooperación y el Desarrollo Económico) ya ha planteado recomendaciones para que los gobiernos conciban fuertes políticas de datos abiertos como instrumentos para contribuir eficazmente a la innovación, el desarrollo en general y muy especialmente a la rendición de cuentas.

Partamos de la definición de los datos abiertos (DA) como una herramienta que facilita en gran medida la transparencia y eficiencia del trabajo, así como el desempeño gubernamental, mediante la reutilización de los datos públicos, incidiendo, como señalan Cruz Rubio y Ramírez Alujas, para dar lugar a nuevas actividades económicas y para asentar la rendición de cuentas, así como para devolver a los ciudadanos los datos que les son propios (Cruz-Rubio, Ramírez 2012). Los Datos Abiertos se entienden como aquellos datos publicados en un formato abierto y estandarizado, que permitan su empleabilidad en servicios y/o aplicaciones útiles para cualquier ciudadano u organización social. De acuerdo con la Open
Knowledge Foundation, son abiertos los datos que garantizan la disponibilidad íntegra a su acceso, la posibilidad de ser redistribuidos, y que fundan en la garantía jurídica el derecho de su uso y reutilización, especialmente aquellos que genera la administración pública. El manual sobre datos abiertos, difundido por la Fundación Open Dat, categoriza a los datos abiertos como disponibles y accesibles, haciendo referencia a la importancia que los datos estén disponibles de modo absoluto y sin costo, y que también sean reutilizables y redistribuibles. En ese mismo sentido, la Carta de Datos Abiertos señala que un requisito infaltable para la gobernanza y la participación ciudadana, es que los datos gubernamentales sean abiertos, por defecto, oportunos, exhaustivos, accesibles, utilizables, comparables e interoperables. Hoffman agrega que los Datos Abiertos no son un componente aislado de la transparencia, sino que además dan la base necesaria para la co-creación entre gobierno y ciudadanos, en un entorno de economía del conocimiento (Hoffman 2015). También Berners-Lee señala que los aspectos jurídicos que permiten el acceso a los Datos Abiertos, se concatenan con políticas deliberadas para la apertura, mediante el uso de estándares abiertos y formatos de archivo no propietarios para la publicación de DA (Berners-Lee, 2006).

Incluso, estos DA son el incentivo para la coproducción, colaboración y participación ciudadana, al proveerles a los funcionarios públicos nuevos insumos de información que impulsen otros enfoques, alternativas 0 propuestas ahora desde la base social (Puron-Cid, et al. 2012).

Afirmamos entonces que el acceso a los DA 
pone a disposición del público conjuntos de datos explotables, que a su vez, al vincularlos con la información procedente de otros conjuntos de datos, facilita el diseño y discusión de nuevos indicadores económicos y sociales para mejorar la toma de decisiones públicas y privadas como ocurre en el caso de la mejora en la gestión del transporte público, el destino de los fondos públicos para la reconstrucción de ciudades o pueblos luego de desastres naturales, entre otras. Varios autores, entre los que se encuentra Alcalde, enumeran algunas clases de DA en función de su uso y aplicación potencial: (a) cultura; (b) ciencia; (c) finanzas; (d) transporte; (e) salud; (f) gobierno; (g) medio ambiente, entre otros (Alcalde, 2017)

Gráfico 2. Los datos abiertos en general y los datos abiertos de medio ambiente.
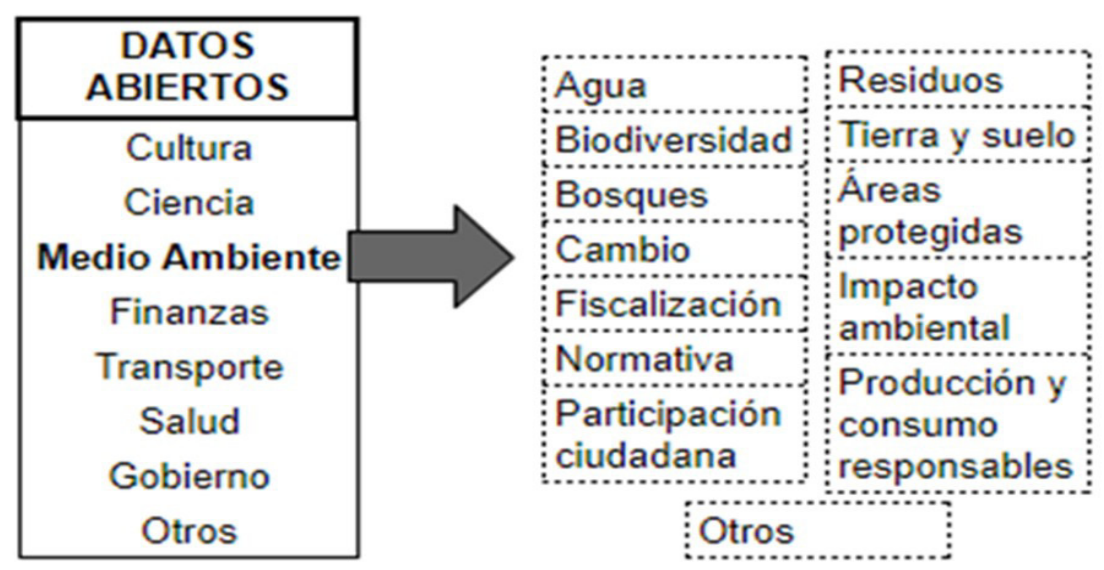

Recuperado de Alcalde, 2017

Otra característica relativa a los DA reside en que estos - incluso aquellos relativos al medio ambiente - se encuentran en bases de datos que ordenan la información en datasets. El término dataset hace referencia a un universo de datos que proporciona un modelo de programación relacional coherente e independiente del origen de los datos que contiene. Los datasets que contienen a los DA se pueden dividir a su vez en diferentes categorías. Por ejemplo, si tomamos en cuenta la clasificación propuesta por la Secretaría de Ambiente y Desarrollo Sustentable del Gobierno Argentino, encontraremos que un dato abierto de medio ambiente (DAMA) podría clasificarse en datasets vinculados con: agua, biodiversidad, bosques, cambio climático, fiscalización, normativa ambiental, impacto ambiental, participación ciudadana ambiental, producción y consumo sustentable, tierra y suelo, residuos, y áreas naturales protegidas, entre otros.

Por lo que respecta a los DAMA, si tomamos los 75 datasets publicados por la Secretaría de Medio Ambiente y Recursos Naturales (SEMARNAT), según la clasificación previamente presentada, observamos que la distribución de los datasets es desigual. Se ha publicado mucho sobre residuos, normativa ambiental y cambio climático (36 datasets en total), y muy poco sobre agua, participación ciudadana y producción sustentable (7 datasets en total). 
HACIA UN DISEÑO DE POLÍtICAS PÚBLICAS DE DATOS ABIERTOS DE MEDIO AMBIENTE

Gráfico 3. Datasets publicados en el portal de datos abiertos por la SEMARNAT

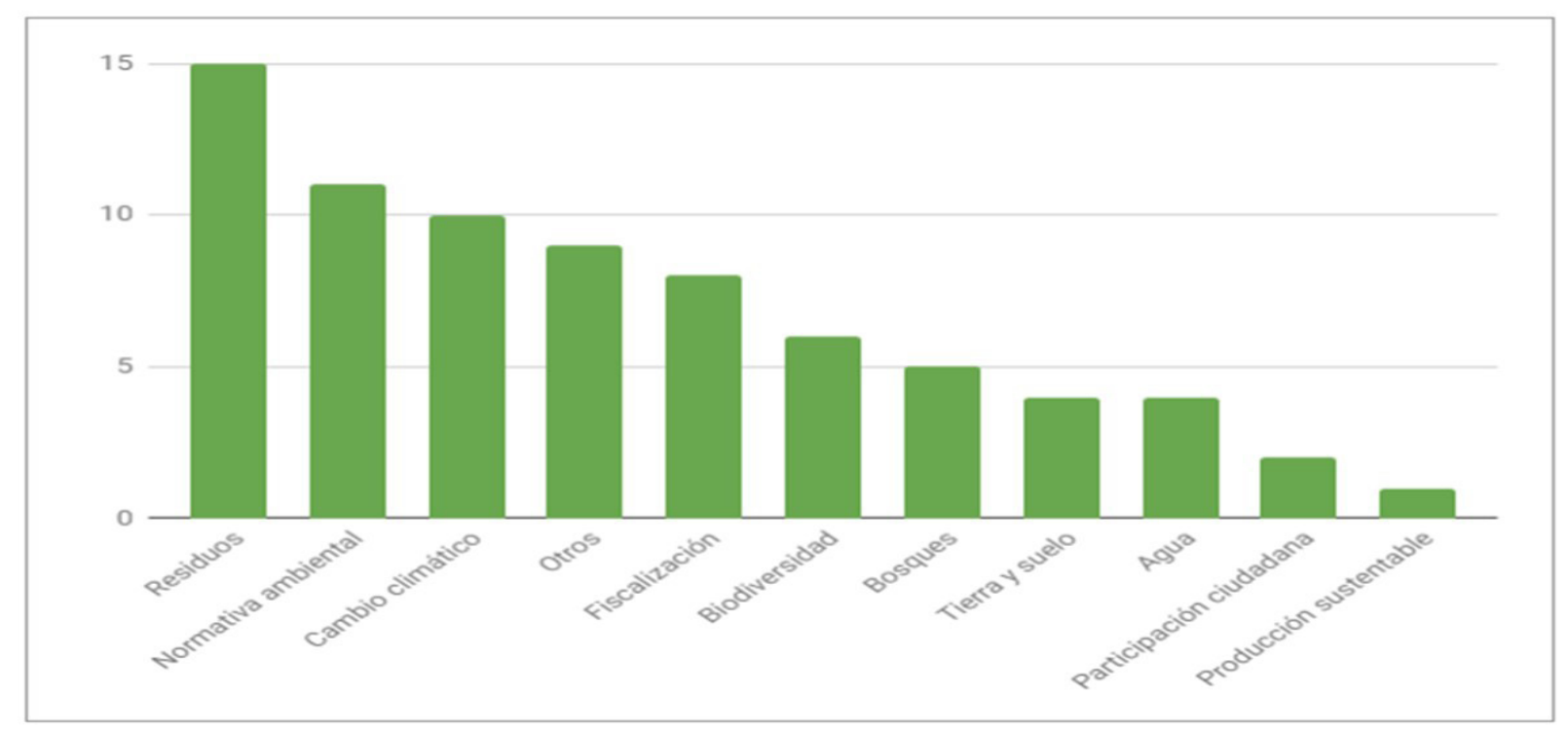

Recuperado de: SEMARNAT, 2018

Por otra parte, existen por lo menos dos índices internacionales que han evaluado a México en el porcentaje de apertura de ciertos DAMA, a saber, el Barómetro de Datos
Abiertos y el Índice Global de Datos Abiertos. En la siguiente tabla se presentan los DAMA evaluados por ambos instrumentos:

Tabla 1. Variables y porcentaje de apertura de datos de medio ambiente en México

\begin{tabular}{l|l|l|l|l}
\hline Año & $\begin{array}{l}\text { Calidad del } \\
\text { Agua }\end{array}$ & $\begin{array}{l}\text { Emisiones Conta- } \\
\text { minantes }\end{array}$ & $\begin{array}{l}\text { Calidad del } \\
\text { Aire }\end{array}$ & $\begin{array}{l}\text { Estadísticas nacionales de } \\
\text { medio ambiente }\end{array}$ \\
\hline 2013 & - & $60 \%$ & - & $60 \%$ \\
2014 & - & $60 \%$ & - & $65 \%$ \\
2015 & $20 \%$ & $45 \%$ & - & $75 \%$ \\
2016 & $0 \%$ & - & $85 \%$ & $60 \%$ \\
2017 & - & - & - & $55 \%$ \\
\hline
\end{tabular}

Fuente: Elaboración propia con base en Barómetro de Datos Abiertos y el Índice Global de Datos Abiertos.

La importancia de los DAMA ha sido también resaltada por la Alianza para el Gobierno Abierto, la cual señaló que la divulgación de tales datos fomenta el acceso público a la información, permite el monitoreo comunitario y exige remedios o alivios en aspec- tos naturales o de la salud.

Incluso, la apertura de los datos ha ocupado un lugar significativo en los tres planes de acción del gobierno mexicano (en 2011, 2013 y 2016 respectivamente) presentados 
a la comunidad internacional en el marco de las acciones de gobierno abierto. En este contexto, el gobierno mexicano se ha comprometido a facilitar el acceso a la informa- ción pública relacionada con ciertos DAMA en diferentes iniciativas, como se detalla en la tabla 2.

Tabla 2. Los datos abiertos de medio ambiente en los planes de acción de gobierno abierto mexicano para la Alianza de Gobierno Abierto entre 2011 y 2018.

\begin{tabular}{|c|c|c|c|}
\hline Nombre de la iniciativa & $\begin{array}{l}\text { Plan de } \\
\text { acción de } \\
\text { Gobierno } \\
\text { Abierto }\end{array}$ & $\begin{array}{l}\text { Instituciones de gobierno res- } \\
\text { ponsables }\end{array}$ & $\begin{array}{l}\text { Tipo de dato de } \\
\text { medio ambiente } \\
\text { vinculado con la } \\
\text { iniciativa }\end{array}$ \\
\hline $\begin{array}{l}\text { Rendición de cuentas } \\
\text { corporativa C: Plástico } \\
\text { PET }\end{array}$ & $\begin{array}{l}\text { Primer plan } \\
(2011-2013)\end{array}$ & $\begin{array}{l}\text { Secretaría de Medio Ambiente } \\
\text { y Recursos Naturales (SE- } \\
\text { MARNAT) }\end{array}$ & Residuos \\
\hline $\begin{array}{l}\text { Rendición de cuentas } \\
\text { corporativa D: Adhesión } \\
\text { de México a EITI. }\end{array}$ & $\begin{array}{l}\text { Primer plan } \\
(2011-2013)\end{array}$ & $\begin{array}{l}\text { Secretaría de Energía, Petró- } \\
\text { leos Mexicanos, Secretaría de } \\
\text { Hacienda y Crédito } \\
\text { Público, Secretaría de Econo- } \\
\text { mía y Secretaría de Relacio- } \\
\text { nes Exteriores }\end{array}$ & $\begin{array}{l}\text { Explotación de } \\
\text { hidrocarburos y } \\
\text { minería }\end{array}$ \\
\hline $\begin{array}{l}\text { Base de datos sobre } \\
\text { proyectos de las com- } \\
\text { pañías mineras }\end{array}$ & $\begin{array}{l}\text { Primer plan } \\
(2011-2013)\end{array}$ & Secretaría de Economía & Minería \\
\hline $\begin{array}{l}\text { Acceso a los estudios } \\
\text { de impacto ambiental }\end{array}$ & $\begin{array}{l}\text { Primer plan } \\
(2011-2013)\end{array}$ & $\begin{array}{l}\text { Secretaría de Medio Ambiente } \\
\text { y Recursos Naturales (SE- } \\
\text { MARNAT) }\end{array}$ & $\begin{array}{l}\text { Informes de im- } \\
\text { pacto ambiental }\end{array}$ \\
\hline $\begin{array}{l}\text { Gastos y financiamiento } \\
\text { del cambio climático }\end{array}$ & $\begin{array}{l}\text { Primer plan } \\
(2011-2013)\end{array}$ & $\begin{array}{l}\text { Secretaría de la Función Pú- } \\
\text { blica, Secretaria de Hacienda } \\
\text { y Crédito Público, y Secretaría } \\
\text { del Medio Ambiente y Recur- } \\
\text { sos Naturales }\end{array}$ & Cambio climático \\
\hline FONDEN transparente & $\begin{array}{l}\text { Segundo } \\
\text { plan (2013- } \\
2015)\end{array}$ & $\begin{array}{l}\text { Secretaría de Hacienda y Cré- } \\
\text { dito Público }\end{array}$ & $\begin{array}{l}\text { Desastres natu- } \\
\text { rales }\end{array}$ \\
\hline $\begin{array}{l}\text { Petróleo en beneficio de } \\
\text { todos }\end{array}$ & \begin{tabular}{|l|} 
Segundo \\
plan $(2013-$ \\
$2015)$ \\
\end{tabular} & Secretaría de Energía & $\begin{array}{l}\text { Explotación de } \\
\text { hidrocarburos }\end{array}$ \\
\hline Minería para todos & $\begin{array}{l}\text { Segundo } \\
\text { plan (2013- } \\
2015)\end{array}$ & Secretaría de Energía & Minería \\
\hline
\end{tabular}


HACIA UN DISEÑO DE POLÍTICAS PÚBLICAS DE DATOS ABIERTOS DE MEDIO AMBIENTE

\begin{tabular}{|l|l|l|l|}
\hline $\begin{array}{l}\text { Cuidado al medio am- } \\
\text { biente basado en evi- } \\
\text { dencia }\end{array}$ & $\begin{array}{l}\text { Segundo } \\
\text { plan (2013- } \\
\text { 2015) }\end{array}$ & $\begin{array}{l}\text { Secretaría de Medio Ambiente } \\
\text { y Recursos Naturales (SE- } \\
\text { MARNAT) }\end{array}$ & $\begin{array}{l}\text { Actualización y } \\
\text { mejora del Siste- } \\
\text { ma Nacional de } \\
\text { Información Am- } \\
\text { biental y de Re- } \\
\text { cursos Naturales } \\
\text { (SNIARM) }\end{array}$ \\
\hline $\begin{array}{l}\text { Protección participativa } \\
\text { del medio ambiente }\end{array}$ & $\begin{array}{l}\text { Segundo } \\
\text { plan (2013- } \\
2015)\end{array}$ & $\begin{array}{l}\text { Secretaría de Medio Ambiente } \\
\text { y Recursos Naturales (SE- } \\
\text { MARNAT) }\end{array}$ & $\begin{array}{l}\text { Participación ciu- } \\
\text { dadana. Informes } \\
\text { de impacto am- } \\
\text { biental }\end{array}$ \\
\hline $\begin{array}{l}\text { Adhesión a la iniciativa } \\
\text { de Transparencia de las } \\
\text { industrias extractivas } \\
\text { (EITI) }\end{array}$ & $\begin{array}{l}\text { Segundo } \\
\text { plan (2013- } \\
2015)\end{array}$ & Secretaría de Energía & $\begin{array}{l}\text { Explotación de } \\
\text { hidrocarburos y } \\
\text { minería }\end{array}$ \\
\hline $\begin{array}{l}\text { Sistema Integral de } \\
\text { información sobre volú- } \\
\text { menes extraídos y con- } \\
\text { cesionado de aprove- } \\
\text { chamiento y descargas } \\
\text { de agua }\end{array}$ & $\begin{array}{l}\text { Tercer plan } \\
(2016-2018)\end{array}$ & $\begin{array}{l}\text { Comisión Nacional del Agua } \\
\text { (CONAGUA) }\end{array}$ & Agua \\
\hline $\begin{array}{l}\text { Reducir la vulnerabili- } \\
\text { dad y riesgos asociados } \\
\text { a los efectos del cambio } \\
\text { climático }\end{array}$ & $\begin{array}{l}\text { Tercer plan } \\
(2016-2018)\end{array}$ & $\begin{array}{l}\text { Secretaría de Medio Ambiente } \\
\text { y Recursos Naturales (SE- } \\
\text { MARNAT) }\end{array}$ & $\begin{array}{l}\text { Desastres natura- } \\
\text { les. Cambio climá- } \\
\text { tico. }\end{array}$ \\
\hline
\end{tabular}

Fuente: Elaboración propia con base en los planes de acción de gobierno abierto mexicano para la Alianza de Gobierno Abierto entre 2011 y 2018. 
Tabla 3. Los datos abiertos de medio ambiente en los planes de acción de gobierno abierto mexicano para la Alianza de Gobierno Abierto entre 2011 y 2018 (en números y porcentual).

\begin{tabular}{l|l|l|l}
\hline $\begin{array}{l}\text { Plan de acción de Go- } \\
\text { bierno Abierto }\end{array}$ & $\begin{array}{l}\text { Compromisos } \\
\text { Totales }\end{array}$ & $\begin{array}{l}\text { Compromisos relacionados } \\
\text { con datos de medio am- } \\
\text { biente }\end{array}$ & En porcentaje \\
\hline $\begin{array}{l}\text { Primer plan (2011-2013) } \\
\text { Segundo Plan (2013- }\end{array}$ & 37 & 5 & $13.5 \%$ \\
$2015)$ & 6 & $23 \%$ \\
Tercer Plan (2016-2018) & 11 & 2 & $18 \%$ \\
Total & 74 & 13 & $17.6 \%$ \\
\hline
\end{tabular}

Fuente: Elaboración propia con base en los planes de acción de gobierno abierto mexicano para la Alianza de Gobierno Abierto entre 2011 y 2018.

A partir de los datos que surgen de las diferentes acciones esgrimidas, podemos observar que la apertura de los DAMA ha constituido un eje central no solo en todos los planes de acción de gobierno abierto mexicano (representando un total de casi $20 \%$ respecto del total de los compromisos asumidos) sino también para la generación de diferentes iniciativas gubernamentales relacionadas con ellos.

Según lo reseñado, observamos que los DAMA son una herramienta que empieza a ser tomada en consideración por México en diferentes áreas de gobierno.

Veremos a continuación si tales datos han sido efectivamente incorporados en lo que consideramos las dos políticas públicas más vinculadas a los mismos, esto es, las políticas públicas ambientales y las políticas públicas de datos abiertos.

Políticas públicas ambientales y políticas públicas de datos abiertos.
Reiteramos, para efectos cognitivos, que la disciplina de políticas públicas ofrece múltiples variantes para definirlas. Afirman Repetto y Moro que "la política pública es una categoría laxa, definida en función del contexto socio histórico que, como concepto, carece de una definición exacta" (Moro y Repetto, 2005). A su vez, Eugenio Lahera ha señalado que la política pública conjunta líneas de acción y flujos de información en torno a un objetivo político diseñado en forma democrática y con el acompañamiento colectivo (Lahera, 2004). Carballeda coincide en ello y plantea que se desprenden de la necesidad de producir una intervención social del Estado, que se presenta como una verdadera "arena" política, que crea y recrea continuamente estos mecanismos de la acción pública (Carballeda, 2002). En ese sentido, la aportación de Chachagua, que también la plantea como una forma de intervención por parte del Estado en lo social, bajo las cuales busca incidir en la fenomenología social, a la luz de un "deber ser" para lograr una transformación sustantiva de 


\section{HACIA UN DISEÑO DE POLÍTICAS PÚBLICAS DE DATOS ABIERTOS DE MEDIO AMBIENTE}

la realidad. En correlación con esto, Bauman entiende a las políticas públicas como respuestas específicas, dirigidas a lo que se entiende, en un contexto y momento determinado como asuntos públicos (Bauman, 2004). Finalmente, podemos referenciar a Cruz-Rubio y Ramirez Alujas para entenderlas como un conjunto articulado, complejo y coherente de ideas, justificaciones, fines, objetivos, recursos, herramientas, disposiciones legales $y / 0$ pronunciamientos que se crean y administran para buscar fines estimados consensualmente como valiosos (Cruz-Rubio, Ramírez, 2012).

Las políticas públicas ambientales (PPA), según Pajares, procuran garantizar la sustentabilidad del desarrollo, con propósitos y acciones de corto, mediano y largo plazos (Parajes, 2008). Según diversos autores (Florino, 1995; OCDE, 2017; Janicke y Weidner, 1997) este tipo de políticas pueden tener diversos objetivos como "(i) la protección de un determinado ecosistema; (ii) el fortalecimiento de la capacidad de ciertos actores; (iii) incidir en factores del contexto como la generación y apropiación social de la información; (iv) la transformación de ciertas condiciones político-institucionales", como la mejora de la coordinación entre diferentes sectores o el fomento de la participación ciudadana, además de mejores condiciones socioeconómicas o tecnológicas.

Desde hace tiempo, muchos países se han preocupado por medir su desempeño en diversas áreas; los datos abiertos en materia medioambiental no son la excepción. Dos de los principales indicadores son: el open data barómetro (2017), que se encarga de evaluar la calidad de las políticas públicas de datos abiertos en 29 países, entre ellos México, analizando diversos sectores, entre ellos el ambiental. Algunos países que sobresalen en esta categoría son Alemania, Reino Unido, Francia, Corea del Sur e Italia. El open data barómetro califica en semaforización, respondiendo 10 preguntas, entre las que destacan la existencia de los datos, disponibilidad, gratuidad, legibilidad y reutilización de los mismos.

De forma similar el Índice de Desempeño Ambiental (Environmental Performance Index, EPI), el cual es un método para cuantificar y clasificar numéricamente el desempeño ambiental de las políticas de un país, utilizando para ello indicadores orientados hacia resultados, sirve como índice de comparación, permitiendo así un mejor entendimiento por parte de políticos, científicos, defensores del ambiente y el público en general; clasifica a 180 países en 24 indicadores de desempeño en diez categorías de temas que cubren la salud ambiental y la vitalidad del ecosistema (EPI, 2018). Los mejores países en el mundo según el EPI 2018 son: Suiza, Francia, Dinamarca, Malta y Suecia.

Sin ánimo de ser exhaustivos, a continuación presentamos en la tabla 4 una breve cronología de las principales acciones gubernamentales relacionadas con las PPA de las últimas décadas en México: 
Tabla 4. Cronología de la política pública ambiental en México.

\begin{tabular}{|l|l|}
\hline 1971 & Ley Federal para Prevenir la Contaminación Ambiental \\
\hline 1972 & Creación de la Subsecretaría de Medio Ambiente (SSMA) \\
\hline 1982 & Ley Federal de Protección del Ambiente \\
\hline 1982 & $\begin{array}{l}\text { Reglamento para la Protección del Ambiente contra la Contaminación por la } \\
\text { Emisión de Ruidos }\end{array}$ \\
\hline 1983 & Creación de la Secretaría de Desarrollo Urbano y Ecología (Sedue) \\
\hline 1983 & $\begin{array}{l}\text { Programa Nacional de Desarrollo 1983-1988 (contiene un apartado sobre ecolo- } \\
\text { gía) }\end{array}$ \\
\hline 1984 & Plan Nacional de Ecología 1984-1988 (PNE) \\
\hline 1985 & Creación de la Comisión Nacional de Ecología (Conade) \\
\hline 1987 & $\begin{array}{l}\text { Reforma constitucional modifica artículos relacionados con el medio ambiente: } \\
\text { Art. 27 y art. 73 }\end{array}$ \\
\hline 1988 & Ley General del Equilibrio Ecológico y Protección al Ambiente (Lgeepa) \\
\hline 1992 & $\begin{array}{l}\text { Creación de la Secretaría de Desarrollo Social (Sedesol), el Instituto Nacional } \\
\text { de Ecología (INE) y la Procuraduría Federal de Protección al Ambiente (Profepa) }\end{array}$ \\
\hline 1992 & $\begin{array}{l}\text { Creación de la Comisión Nacional para el Conocimiento y Uso de la Biodiversi- } \\
\text { dad (Conabio) }\end{array}$ \\
\hline 1994 & Secretaría del Medio Ambiente, Recursos Naturales y Pesca (Semarnap) \\
\hline 1994 & $\begin{array}{l}\text { Reforma importante de la Ley General del Equilibrio Ecológico y Protección al } \\
\text { Ambiente (Lgeepa) }\end{array}$ \\
\hline 1998 & Reforma constitucional \\
\hline 2000 & Ley General de la Vida Silvestre \\
\hline 2001 & Secretaría de Medio Ambiente y Recursos Naturales (Semarnat) \\
\hline 2001 & $\begin{array}{l}\text { Reforma de la Ley General del Equilibrio Ecológico y Protección al Ambiente } \\
\text { (Lgeepa) }\end{array}$ \\
\hline 2001 & Programa para Promover el Desarrollo Sustentable en el Gobierno Federal \\
\hline 2003 & Ley General del Desarrollo Forestal Sustentable \\
\hline 2003 & Ley General para la Prevención y Gestión Integral de los Residuos \\
\hline 2004 & Ley de Aguas Nacionales \\
\hline 2005 & Ley de Bioseguridad \\
\hline 2006 & Ley de Productos Orgánicos \\
\hline 2007 & $\begin{array}{l}\text { Reformas de la Ley General del Equilibrio Ecológico y Protección al Ambiente } \\
\text { Lgeepa) }\end{array}$ \\
\hline 2007 & Plan Nacional de Desarrollo (PND) \\
\hline 2008 & $\begin{array}{l}\text { Reforma de la Ley General del Equilibrio Ecológico y Protección al Ambiente } \\
\text { Lgeepa) }\end{array}$ \\
\hline
\end{tabular}


Si estudiamos cada una de estas acciones a la luz de lo enunciado por Guevara podemos vislumbrar que dentro de estas acciones conviven, en mayor o menor medida, tres tipos de instrumentos en materia de PPA: (a) normativos, que buscan regular el comportamiento ambiental desde una visión normativa; (b) educativos, que pretenden concientizar y formar en materia ambiental, para prevenir y transformar ciertas conductas; y (c) económicos, los cuales utilizan mecanismos que intervienen en la racionalidad económica de los actores, para incentivarlos a corregir las externalidades negativas sobre el ambiente (Guevara , 2007).

Pensemos que las políticas públicas de da- tos abiertos (PPDA) han sido definidas por la doctrina, de una forma más amplia que la de ejercer política pública "pura" (Prince et al., 2013) abarcando también decisiones políticas fruto de la sociedad de la información, vinculadas normalmente con la tecnología y los datos, alcanzando un efecto o beneficio social directo o indirecto, que trastoca toda suerte de relaciones e intercambios. Así, una PPDA es aquella que es capaz de identificar y generar acciones concretas que permitan resaltar el real valor de los DA, esto es, generar interés y acciones que permitan la reutilización de tales datos. La siguiente tabla 5 presenta las principales acciones relacionadas con las PPDA en México:

Tabla 5. Evolución de las políticas públicas de datos abiertos en México.

\begin{tabular}{|l|l|}
\hline 1997 & Reforma constitucional reconoce derecho a la información (Art. 6) \\
\hline 2001 & $\begin{array}{l}\text { Plan Nacional de Desarrollo 2001-2006 reconoce como objetivo construir un go- } \\
\text { bierno abierto y transparente }\end{array}$ \\
\hline 2002 & Ley Federal de Transparencia y Acceso a la Información Pública Gubernamental \\
\hline 2002 & Creación del Instituto Federal de Acceso a la Información Pública \\
\hline 2007 & $\begin{array}{l}\text { Constituyente Permanente aprueba estándares mínimos para acceso a la infor- } \\
\text { mación, datos personales y derecho de rectificación. }\end{array}$ \\
\hline 2009 & Se crean órganos garantes de acceso a la información \\
\hline 2010 & Creación Instituto Federal de Acceso a la Información y Protección de Datos \\
\hline 2012 & México integra la Alianza para el Gobierno Abierto \\
\hline 2012 & Primer plan de acción de gobierno abierto de México \\
\hline 2013 & $\begin{array}{l}\text { Estrategia Digital Nacional 2013-2018 para fomentar la adopción y el desarrollo de } \\
\text { los datos abiertos }\end{array}$ \\
\hline 2013 & $\begin{array}{l}\text { Publicación de las Bases de Colaboración: lineamientos y metodología para la li- } \\
\text { beración de grupos de datos abiertos }\end{array}$ \\
\hline 2013 & Creación de la Dirección General de datos abiertos \\
\hline 2013 & Segundo plan de acción de gobierno abierto de México \\
\hline 2014 & Grupo Escuadrón de Datos \\
\hline 2014 & $\begin{array}{l}\text { Se provee autonomía constitucional al Instituto Federal de Acceso a la Informaci- } \\
\text { ón y Protección de Datos }\end{array}$ \\
\hline 2015 & Política Nacional de Datos Abiertos \\
\hline
\end{tabular}




\begin{tabular}{|l|l|}
\hline 2015 & Decreto (DOF: 20/02/2015) establece la regulación en materia de Datos Abiertos \\
\hline 2015 & Publicación de la Guía de Implementación de la Política de Datos Abiertos \\
\hline 2015 & Red México Abierto \\
\hline 2016 & Labora: iniciativa de desarrollo económico con datos abiertos \\
\hline 2016 & Tercer plan de acción de gobierno abierto de México \\
\hline
\end{tabular}

Entendemos que las PPDA pueden ser simples o plenas, según el cumplimiento de sus diferentes fases de implementación. Esta clasificación permite conocer las acciones en ejecución o pendientes de cada una de las fases de una PPDA determinada y diferenciarla. Así una PPDA simple es aquella que no ha completado todas sus fases de implementación, a saber: a) la fase de acceso a la información, b) la fase de apertura de datos, c) la fase de reutilización, y d) una fase de evaluación, complementaria a las enunciadas. Una PPDA plena es aquella que cumple con cada una de las fases citadas. Creemos que para que una PPDA sea plena y pueda cumplir con sus fines debe someterse a evaluación, a fin de poder identificar oportunidades, corregir errores y/o incorporar acciones no pensadas al momento inicial de su diseño. Cabe destacar que México aún tiene pendiente la elaboración de su propia herramienta de evaluación y hasta la fecha solo ofrece información integrada en diferentes índices que, no obstante, han arrojado resultados constructivos en diferentes campos de actividad.

En contraste, subrayamos que las PPDA constituyen una manera diferente de ejercer un gobierno que integre decisiones y acciones políticas, planteándose el logro de transformaciones por medio de la redefinición de la relación gobierno-datos-ciudadano, teniendo como fin último el empoderamiento de su colectivo de ciudadanos.

\section{DISCUSIÓN DE RESULTADOS}

Buscando una nueva dimensión de políticas públicas: las políticas públicas de datos abiertos de medio ambiente

Como hemos destacado en (1) los DAMA son uno de los datos centrales al momento de considerar acciones de apertura de datos, tanto a nivel nacional como internacional. También se ha enfatizado que (2) las políticas públicas se erigen como una herramienta de intervención social, orientadas a satisfacer necesidades colectivas, atendiendo la agenda socialmente construida, en donde los órganos ejecutivos o representativos estatales intervienen deliberadamente con la intención de corregir, modificar o mejorar una situación específica en el seno de la sociedad, que ha sido reconocida como problema atendible públicamente. Esta decisión aperturista que se ha desarrollado en las últimas dos décadas tuvo a la transparencia como concepto central (Elster, 1998) donde transparentar la gestión pública (Ontiveros, 2004) implica consolidar la rendición de cuentas a los ciudadanos, los cuales tienen a su alcance el derecho humano a demandar información precisa, confiable y comprobable de los actos y decisiones del gobierno (Doyle, 2003). Podemos resaltar entonces que, en este contexto, las PPDA se basan en la idea de transparentar las actividades gubernamentales, poniendo al alcance de la sociedad informa- 


\section{HACIA UN DISEÑO DE POLÍTICAS PÚBLICAS DE DATOS ABIERTOS DE MEDIO AMBIENTE}

ción y datos públicos.

Como señala Vera (2018):

"En los últimos años hemos asistido a un... proceso cultural de empoderamiento colectivo de acceso a los datos. La información generada por organismos públicos, debido a su abundancia y calidad... debe estar al servicio de todos los ciudadanos".

En el mismo artículo se cita a Lathrop y Ruma, sosteniendo que "la información pública se ha convertido en una forma de infraestructura con el mismo nivel de importancia que la del agua, la electricidad o las carreteras. En tal sentido es necesario maximizar el valor público que los datos tienen para la transparencia y la innovación" (Lathrop y Ruma, 2010).

En ese sentido, Santoyo Serrano \& Martínez, afirman que la sociedad en red, definitoria del Siglo XXI, ha tenido como resultado significativo el surgimiento de la era de la información, en donde la digitalización de la sociedad es creciente y cada vez más global, lo que produce nuevas variantes de interacción (Serrano Santoyo y Martinez, 2003). Sin embargo, hemos observado que, hasta la fecha, las PPA no reflejan claramente a los DA como parte del diagrama de sus acciones. Por el contrario, los mismos se encuentran disponibles para consulta, pero sin encontrarse debidamente integrados dentro de una PPA acorde que aproveche su utilización y el valor que aportan. En igual sentido, también hemos observado que las PPDA, si bien los han integrado dentro de sus acciones aperturistas, no han implementado en el diseño y ejecución de sus fases de implementación, acciones coordinadas de reutilización y/o evaluación de los DAMA.

En general los datos abiertos en gobierno, aún tienen diversos desafíos, entre los cuales Velázquez destaca: Sobrevivir a los cambios de administración, para ello es importante contar con una ley de datos abiertos, ya que en la actualidad existen documentos como el Decreto de Datos Abiertos, la Guía de implementación de la Política de Datos Abiertos, el Índice de Datos Abiertos establecido por el Programa para un Gobierno Cercano y Moderno (PGCM) 2013-2018 y la Norma Técnica para el Acceso y Publicación de Datos Abiertos de la Información Estadística y Geográfica de Interés Nacional, entre otros (Velázquez, 2017). Estos documentos, al ser parte de una normativa administrativa -y no una ley como tal- son también susceptibles de derogarse en cualquier momento. Ligado a este punto, otra debilidad de los DA en México es la estandarización y calidad de los datos, ya que existen discrepancias en las prácticas de datos abiertos que se realizan entre la federación, los estados y los municipios, porque no hay forma de obligarlos a estandarizarlas, por ello es necesario establecer reglas de gobierno sobre la difusión e intercambio de datos, especialmente cuando incluyen información personal sensible, así como aumentar la eficiencia de los servicios prestados por las entidades federales y los municipios respecto al nivel de calidad, velocidad de entrega, simplificación de los procedimientos y reducción de los costos operativos.

También hay que prever sanciones en caso de difusión de datos que contengan errores. Por otro lado, es importante que tanto ciuda- 
danos como otros actores sociales participen en la creación de capacidades digitales.

En ese tenor, es vital asegurarse de realizar una adecuada clasificación de la información que compone las bases de datos, delineando lo que "debe ser público" con base en la Ley Federal de Transparencia y Acceso a la Información Pública, y lo que "debe ser resguardado" como parte del cumplimiento de la Ley Federal de Protección de los Datos Personales en Posesión de Particulares (LFPDPPP). Para ello, lo importante es que los equipos de apertura de datos cuenten con un experto en privacidad y otro en transparencia. Finalmente, el área de mejora más importante es la ética en la publicación de los datos; la mayoría de los gobiernos inicia con la publicación de información que es relativamente "fácil" de revelar en términos técnicos o de contenido. Pero es perfectamente posible que la transparencia conviva con la corrupción, ya que la información publicada no toca los puntos vulnerables de la organización. Esta entrega de "información a medias" llegar a ser incluso más dañina porque genera desconfianza de la información ya publicada y puede echar por la borda los esfuerzos de apertura de datos. Así que el verdadero reto está en lograr que el gobierno publique, gradualmente y de forma proactiva, información gubernamental, aunque esta no sea tan "favorecedora" para el gobierno en turno.

Es así que identificamos que tanto las PPA (sean explícitas o implícitas) como las PPDA (sean simples o plenas) carecen de un sistema de articulación o coordinación conjunto en el tratamiento de los DAMA.

En este contexto de análisis, consideramos que tanto las políticas ambientales como las políticas de datos abiertos necesitan no sólo ser complementarias entre sí para ser eficientes y efectivas sino también necesitan integrar al dato abierto de medio ambiente como un elemento singular dentro de su diseño para obtener un real impacto positivo y promover el desarrollo sustentable

Este efecto de complementariedad, da lugar a un subtipo de política pública, el cual denominamos política pública de datos abiertos de medio ambiente. 
HACIA UN DISEÑO DE POLÍtICAS PÚBLICAS DE DATOS ABIERTOS DE MEDIO AMBIENTE

Gráfico 4. Esquema de conformación de una política pública de datos abiertos de medio ambiente.

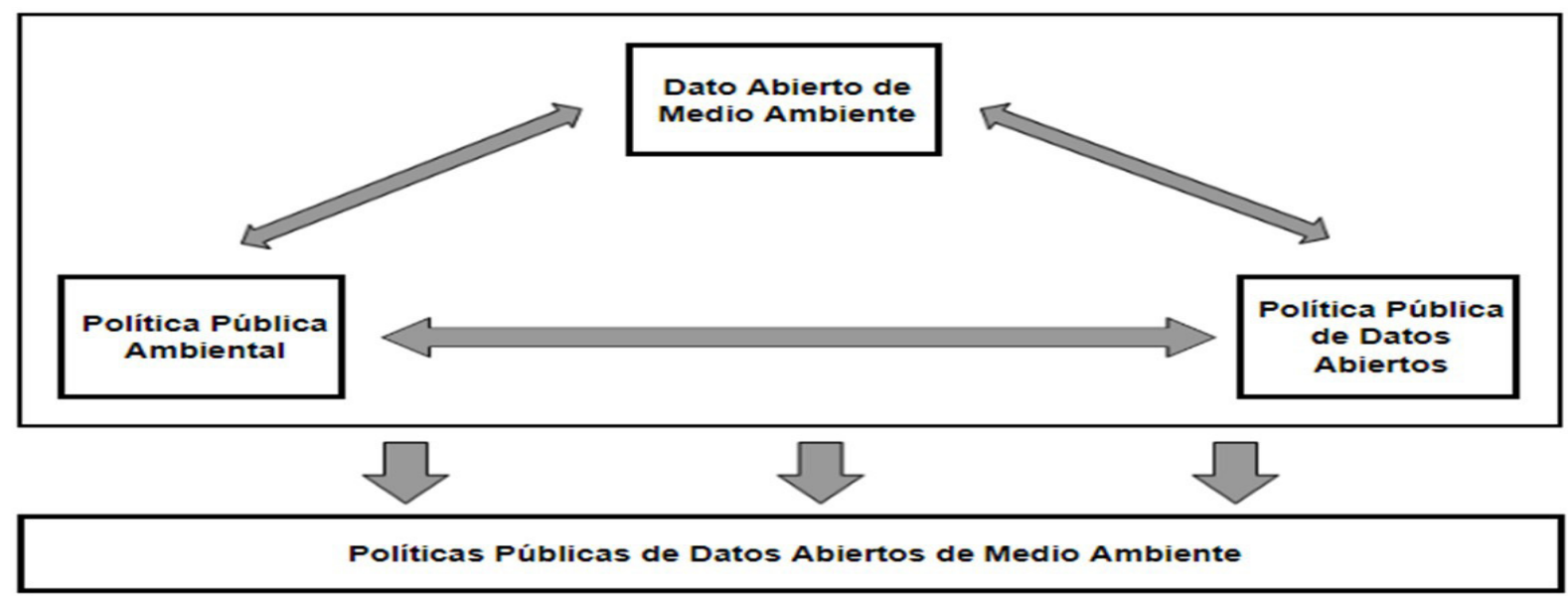

Una política pública de datos abiertos de medio ambiente (PPDAMA) es aquella que se vale del dato abierto de medio ambiente y que tiene por finalidad promover su acceso y reutilización, conjuntamente con la evaluación de cada una de las fases que la integran. En este contexto, reiteramos la definición ya presentada de dato abierto de medio ambiente; es todo dato relacionado con el medio ambiente y presentado en un formato abierto que permite su acceso redistribución y reutilización por parte de cualquier persona. Las PPDAMA se presentan como una herramienta eficaz y eficiente tanto para los ciudadanos como para el propio Estado en sus acciones de políticas públicas ambientales. La complementariedad entre los diferentes elementos destacados en el gráfico 4 nos permite ilustrar tres tipos de variantes que constituyen una PPDAMA. Así, este tipo de política será resultado de:

a. La inclusión de los DAMA en las políticas públicas ambientales. Esto permite una reutilización singular dentro del marco de este tipo de políticas. b. La inclusión de los mecanismos de PPDA en las políticas públicas ambientales.

c. La inclusión de los mecanismos de las políticas ambientales en el diseño de las PPDA, teniendo como eje diferenciado el DAMA. Las políticas ambientales tienen rasgos singulares, generalmente no tomados en cuenta al momento de trabajar con datos abiertos.

Como resultado de la interacción representada, las PPDAMA tienen ciertas características especiales y diferenciadoras, a saber:

1. Su eje es el DAMA. Este puede ser obtenido ya sea de iniciativas dentro de las políticas públicas ambientales, de las PPDA o bien de acciones privadas o de gobierno ajenas a una política pública específica.

2. Normativa específica. Requieren de instrumentos normativos específicos que permitan el acceso a todos los datos de medio ambiente de carácter 
público y, de ser posible, también privados.

3. Estructura organizativa múltiple. Requieren una o más instituciones que, de manera coordinada ejecuten la implementación de sus diferentes procedimientos, criterios de decisión y programas.

4. Favorecen la transparencia ambiental.

Hilda Naessens aporta la idea de que la transparencia es un atributo o cualidad que permite contar con más información, de manera clara y precisa sobre una persona o asunto, lo que produce el aumento de nuestras capacidades de comprensión, vigilancia y comunicación (Naessens, 2010). Las PPDAMA apuntalan la transparencia de la información ambiental y la búsqueda de los ciudadanos, por parte de los mercados y de los gobiernos, para que sus políticas y sus resultados, se sustenten en instituciones cada vez más legítimas y creíbles.

5. Permiten generar acciones concretas para el reúso social de los DAMA. El valor pleno de las PPDAMA se basa, como en toda PPDA, en dicha reutilización de los datos abiertos. Los DAMA, por su complejidad y características propias, tienen mecanismos propios de reutilización, mismos que para el logro de los fines de la política ambiental, deben ser garantizados como abiertos y accesibles por las instituciones administrativas y legales.

\section{CONCLUSIONES}

Consideramos que los DAMA de México conforman un tipo de DA con sus particu- laridades y efectos singulares y que en tal sentido merecen un tratamiento público diferencial. Tal tratamiento o acción pública surge, según nuestra propuesta, de acciones complementarias entre las políticas públicas ambientales y las PPDA quienes, actuando en conjunto y en colaboración, generarán un tipo de política pública nueva, esto es, la política pública de dato abierto ambiental.

Esta nueva política surge como resultado de una acción conjunta de gobierno, donde los DAMA se verán sometidos a la estructura de las PPDA pero al mismo tiempo tendrán un enfoque ambientalista tal como lo proponen las políticas medioambientales. Consideramos nuestra propuesta como un avance en el debate sobre políticas públicas y en particular pretendemos que las diferentes propuestas vertidas en el presente sirvan como el inicio de nuevos abordajes teóricos de la materia.

La propia SEMARNAT (2018) reconoce que la insustentabilidad de los procesos productivos está generando límites de base en muchos de los ecosistemas naturales. Además, los procesos de deterioro han sido tan severos, que ya resultan desastrosos para varios aspectos de la vida humana o amenazan con serlo. Las primeras manifestaciones de los procesos de deterioro ocurrieron en el ámbito local, después se ampliaron al espacio regional y en la actualidad se extienden por todo el territorio nacional. En estos procesos la temporalidad de sus efectos es un factor clave:

- Algunos de estos procesos como la deforestación, el deterioro de los suelos, la sobreexplotación de los recursos, la sequía y la escasez del agua, 


\section{HACIA UN DISEÑO DE POLÍTICAS PÚBLICAS DE DATOS ABIERTOS DE MEDIO AMBIENTE}

están manifestando sus efectos en el corto plazo.

- Otros, como la capacidad limitada de la atmósfera para absorber las emisiones excesivas de $\mathrm{CO} 2$, la contaminación del agua y los vertidos insustentables de residuos sólidos en ríos y mares, apenas si se muestran en algunas regiones y ciudades del país. Pero sus efectos verdaderos, amenazan con manifestarse intensamente en el mediano y largo plazo.

Las tendencias destructivas para el medio ambiente han aumentado, que no sólo están agotando el capital natural del país y acelerando los procesos de contaminación de aire, agua, suelo, sino que también están frenando las capacidades productivas y repercuten en el ámbito social, los efectos socio-ambientales que se traducen en pérdidas de la producción, particularmente alimentos, puestos de trabajo, caídas en los ingresos de la población y de las exportaciones, aumentos en el número de epidemias, enfermedades y daños a la salud de la población, así como en incremento de las migraciones masivas del campo a la ciudad y de los niveles de marginación y pobreza.

\section{CONSIDERACIONES FINALES}

Este análisis tuvo como fin presentar una aproximación al estudio de las políticas públicas de datos abiertos de medio ambiente en México, teniendo en consideración la complementariedad entre los datos abiertos de medio ambiente ya existentes y las diferentes herramientas que ofrecen las políticas ambientales y de datos abiertos. Es pertinente reiterar que no se trata de analizar si existen datos o sistemas de creación de bases de datos públicos, sino de conceptualizar y ubicar Políticas Públicas de datos Abiertos de Medio Ambiente, que dejan de ser "pasivos", para producir sistemas que realmente impacten el trabajo de gobierno y su uso y reuso en todos los sectores de una nación. Presentamos a las políticas públicas de datos abiertos de medio ambiente como un universo en pleno auge y consolidación, que se caracteriza por objetivos, principios y criterios específicos para el diseño de acciones viables que transformen creativamente la relación de las instituciones públicas con la ciudadanía y su calidad de vida, en el contexto de la necesidad del desarrollo sostenible. Del estudio se desprende que las políticas públicas de datos de medio ambiente, deben ser el resultado lógico de un modelo de interacción entre herramientas políticas ya existentes que deben alcanzar su re-adaptación al contexto contemporáneo de acceso a la información y a la innovación de la tecnología, que abarca prácticamente todos los campos humanos, económicos, administrativos y sociales. El entramado y la orientación de las instituciones de gobierno, precisa de una verdadera reinvención, para que se acerque al logro de su creciente demanda, exposición pública y dinámica global, sin poner en riesgo los fines colectivos que determinan su naturaleza y misión humana.

\section{REFERENCIAS}

Alcalde, I., (2017). Open data: Datos transparencia y conocimiento abierto. s.l.:s.n. Recuperado de https://ignasialcalde.es/ open-data-datos-transparencia-y-conocimiento-abierto/

Barometro, O. D. (2017). Enviro- 
ment performance index. Recuperado de https://opendatabarometer.org/? year $=2017$ \&indicator $=$ ODB

Bauman, Z. (2004). La sociedad sitiada. Buenos Aires: Cúspide.

Carballeda, A.J. (2002). La intervención en lo social: Exclusión e integración en los nuevos escenarios sociales. Buenos Aires: Paídos.

Chachagua, M.R., (2015). Aproximaciones a los usos sociales de las TICs en instituciones educativas: Los docentes y las netbooks del Programa Conectar Igualdad. III Jornadas Nacionales sobre estudios regionales y mercados de trabajo. Universidad Nacional de Jujuy, pp. 5-6.

Cortés-Ontiveros, R. (2005) . La transparencia en México: Razón, Origen y Consecuencias. Revista de la facultad de derecho de México, Vol. 55, No. 244, pp. 13-30. dol: 10.22201/fder.24488933e.2005.244.61566

Cruz-Rubio, C.N. (2015). ¿Qué es (y qué no es) gobierno abierto? Una discusión conceptual. Economía. Revista en cultura de la legalidad, 8,37-53.

Cruz-Rubio,C.N., \& Ramírez Alujas, A.V.(2012). ¿Políticas Públicas Abiertas? Apuntes exploratorios para el análisis y transformación de los diseños políticos bajo los principios del gobierno abierto. Madrid: GIGAPP Estudios Working Papers. Recuperado de: http://www.gigapp.org/ewp/index. php/GIGAPP-EWP/article/view/24

Doyle, K. (2003). Comentarios sobre la Ley Federal de Transparencia y Acceso a la In- formación Pública Gubernamental. En: Derecho comparado de la información . s.I.:s.n., pp. 163-172.

Elster, J., (1998). Deliberation and Constitution Making. En: Deliverative Democracy, pp. 97-118.

EPI Index, E. P., (2018). Yale University. Recuperado de https://epi.envirocenter.yale. edu/2018/report/category/hlt

Espinoza, P., (2018). índice de desempeño ambiental. Calificación del mundo en desarrollo sostenible en el 2018. Recuperado de https://pensandodesdecentroamerica.wordpress.com/2018/02/21/indice-de-desempeno-ambiental-calificacion-del-mundo-en-desarrollo-sostenible-en-el-2018/

Fiorino, D.J. (1995). Making Environmental Policy. University of California Press.

Hoffman, A. (2015). Gobierno abierto y el valor social de la información pública. CDMX: Instituto de investigaciones jurídicas de la UNAM.

Índice Global de Datos Abiertos, (2013). Indice Global de Datos Abiertos. Recuperado de http://2015.index.okfn.org/place/mexico/2013/

Índice Global de Datos Abiertos., (2014). Indice global de datos abiertos. Recuperado de http://2015.index.okfn.org/place/mexico/2014/

Índice Global de Datos Abiertos, (2015). Indice Global de Datos Abiertos. Recuperado de http://2015.index.okfn.org/place/mexico/2015/ 


\section{HACIA UN DISEÑO DE POLÍTICAS PÚBLICAS DE DATOS ABIERTOS DE MEDIO AMBIENTE}

Índice Global de Datos Abiertos, (2016). Indice Global de Datos Abiertos. Recuperado de http://2015.index.okfn.org/place/mexi$\mathrm{co} / 2016 /$

Janicke, M. \& Weidner, H. (1997). National Enviromento Policies. Berlín: Springer.

Lahera, E. (2004). Política y Políticas Públicas. Repositorio de naciones unidas. En: s.l.:s.n., pp. 21-23.

Lathrop, D. \& Ruma, L. (2004). Open Government: Collaboration, Transparency, and Participation in Practice. O'Reilly Media, Sebastopol. s.l.:s.n.

Lezama, J. L., (2010). Sociedad, medio ambiente y política ambiental, 1970-2010, Los grandes problemas de Méxolíticas Púico. s.l.: El Colegio de México.

Lowi, Theodore J.; Allison, Graham T.; Lindblom, Charles E.; Dror, Yehezkel; Etzioni, Amitai; Goodin, Robert; Waldner, IImar; Forester, John; Majone, Giandomenico; Meltzer, Arnold J. (1996.) Pblicas, Estudios de Caso y Teoría Política. En: La hechura de las políticas públicas. s.I.:Porrua.

Machorro, J. C., (2019). México olvida el impacto ambiental del huachicoleo, el robo de combustible. Recuperado de https://www. efeverde.com/noticias/mexico-impacto-ambiental-huachicoleo-robo-combustible/

Martínez, M.C.V. (2018). Los datos abiertos y el Plan de Acción de Gobierno Abierto 2013-2015. Entrevistas UACJ, pp. 1-18.
México, G. d., (2015). Plan de acción del gobierno mexicano. Recuperado de http:// www.pa2015.mx/pa_aga_2015.pdf

Moro, Javier; Repetto, Fabián, (2005). La política como restricción y como oportunidad: alcances y ámbitos de coordinación de la política social. Santiago, Congreso internacional del CLAD.

Naessens, H. (2010). Ética pública y transparencia. En: Rey E. \& González, P.C. (coord.) 200 años de Iberoamérica (1810-2010). España, Congreso internacional actas del XIV Encuentro de latinoamericanistas españoles.

OCDE, (2017). Estudio de la OCDE sobre Datos Abiertos Gubernamentales de México, Estudio de Datos Abiertos Gubernamentales de México, Impacto del sector público e innovación a través del reúso de datos". Recuperado de www.oecd.org/gov/ digital-government/DigGovMex-SummarySpanish.pdf

Open Data Charter., (2017). Open data charter. Recuperado de http://opendatacharter.net/principles/

Open Data Handbook, (2018). Recuperado de http://opendatahandbook.org/

Organización Mundial de la Salud y Global Environment Monitoring System, (1992). Urban air pollution in megacities of the world. s.l.:Blackwell reference.

Oslak, O. (2013). "Gobierno abierto: Hacia un nuevo paradigma de gestión pública". Recuperado de www.oas.org/es/sap/dgpe/ pub/coleccion5RG.pdf 
Parajes, E. (2008). La definición de políticas públicas ambientales y su aplicación en los sistemas territoriales. En: Perú Hoy. Territorio y naturaleza. Desarrollo en armonía. Lima: Desco.

Pérez Calderón, J. (2010). La política ambiental en México: Gestión e instrumentos económicos.. El Cotidiano, pp. 91-97.

Plan de Acción del Gobierno Mexicano, (2011). Recuperado de https://www.opengovpartnership.org/sites/default/files/Mexico_Action_Plan.pdf

Plan de Acción del Gobierno Mexicano, (2015). Recuperado de https://www.opengovpartnership.org/sites/default/files/Mexico_Action_Plan.pdf

Plan de Acción del Gobierno Mexicano, (2016). Recuperado de https://www.opengovpartnership.org/sites/default/files/Mexico_Action_Plan.pdf

Prince A, Jolias, L., \&Brys, C.,R., (2013). Análisis de la cadena de valor del ecosistema de Datos Abiertos de la Ciudad de Buenos Aires. Recuperado de http://www. princeconsulting.biz/pdf/7.pdf

Puron-Cid,G., Gil-García, J.R. \& Luna-Reyes, L.F. (2012). IT-enabled policy analysis: New technologies, sophisticated analysis and open data for better government decisions. 13th Anual international conference on digital goverment, pp. 97-106. doi:10.1145/2307729.2307746

Ramírez-Alujas, A. \& Cruz-Rubio,C.N. ( 2012). Políticas Públicas Abiertas: Hacia la definición y análisis de los diseños políticos bajo los principios del gobierno abierto. Buen Gobierno, pp. 52-76.

Ramírez-Alujas, A. (2011). Gobierno abierto y modernización de la gestión pública: tendencias actuales y el (inevitable) camino que viene. Reflexiones seminales. Revista Enfoques, pp. 99-121.

Rios, K.V. (2017). Promesas y realidades de los datos abiertos. Recuperado de https://ugob.com/promesas-y-realidades-de-los-datos-abiertos/

Sangines, A.G., (2007). Política ambiental en México: Génesis, desarrollo y perspectiva. En: Políticas públicas para un crecimiento incluyente . Mexico: Universidad Iberoamericana , pp. 365-387.

Santoyo, A.S. \& Martinez, E.M. (2003). La brecha digital: Mitos y Realidades. Ed: Universidad Autonoma de Baja California. s.I.:s.n.

Secretaría del Medio Ambiente de la Ciudad de México, SEDEMA, (2016). Secretaría del Medio Ambiente de la Ciudad de México. Calidad del aire en la Ciudad de México, informe 2016, s.l.: Ciudad de México.

Secretaría del Medio Ambiente y Recursos Naturales, SEMARNAT, Gobierno de México. (2018). Desastres y procesos de deterioro ambiental. Recuperado de http://dgeiawf.semarnat.gob.mx:8080/ ibi_apps/WFServlet?IBIF_ex=D1_R_ DESASTRE00_01\&IBIC_user=dgeia_ mce\&IBIC_pass=dgeia_mce

Shadbolt, N.; Berners-Lee, T., Hall, W., (2006). The semantic web revisited. IEEE In- 


\section{HACIA UN DISEÑO DE POLÍTICAS PÚBLICAS DE DATOS ABIERTOS DE MEDIO AMBIENTE}

teligent systems vol. 21 , no. 3 , pp. 96-101, Jan.-Feb. 2006, doi: 10.1109/MIS.2006.62.

World Health Organization \& Global Environment Monitoring System, (1992). Urban air pollution in megacities of the world. s.l.:Oxford.

World Wide Web Foundation (2013). Open Data Barometer Global Report: First Edition. Washington, DC.
World Wide Web Foundation (2015). Open Data Barometer Global Report: Second. Ed: Washington, DC.

World Wide Web Foundation 2016. Open Data Barometer Global Report: Third Ed.: Washington, DC.

World Wide Web Foundation 2017. Open Data Barometer Global Report: Fourth Ed: Washington, DC. 